\title{
Erratum
}

\section{Evaluation of generalized exponential integrals using multinomial expansion theorems}

\author{
B.A. Mamedov, ${ }^{*}$ Z. Merdan and I.M. Askerov \\ Department of Physics, Faculty of Arts and Sciences, Gaziosmanpaşa University, Tokat, Turkey \\ E-mail: bamamedov@yahoo.com
}

Erratum to: Journal of Mathematical Chemistry

DOI: $10.1007 / \mathrm{s} 10910-005-6918-\mathrm{y}$

The author regrets that in the above article equations (4), (10) were some misprints. They are now reproduced correctly below:

$$
\begin{aligned}
\left(x_{1}+x_{2}+x_{3}+\cdots+x_{t}\right)^{n}= & \sum_{n_{1}=0}^{n} \sum_{n_{2}=0}^{n-n_{1}} \sum_{n_{3}=0}^{n-n_{1}-n_{2}} \cdots \sum_{n_{t}=n-n_{1}-n_{2}-\cdots-n_{t-1}}^{n-n_{1}-n_{2}-\cdots-n_{t-1}} \\
& F_{n_{1}, n_{2}, n_{3}, \cdots, n_{t}}(n) x_{1}^{n_{1}} x_{2}^{n_{2}} x_{3}^{n_{3}} \cdots x_{t}^{n_{t}} \\
E_{s}^{n}(x)= & \frac{1}{\Gamma(n+1)} \sum_{n_{1}=0}^{n} \sum_{n_{2}=0}^{n-n_{1}} \sum_{n_{3}=0}^{n-n_{1}-n_{2}} \cdots \sum_{n_{t}=n-n_{1}-n_{2}-\cdots-n_{t-1}}^{n-n_{1}-n_{2}-\cdots-n_{t-1} \sum_{1}+2 n_{2}+\cdots+t n_{t}} \sum_{i=0}^{n} \\
& (-1)^{i} F_{n_{1}, n_{2}, n_{3}, \cdots, n_{t}}(n) F_{i}\left(n_{1}+2 n_{2}+\cdots+t n_{t}\right) \\
& \times \frac{1}{2^{n_{2} 3^{n_{3}} \cdots t^{n}}} E_{i+s}(x)
\end{aligned}
$$

* Corresponding author.

The online version of the original article can be found under doi:10.1007/s10910-005-6918-y 